\title{
Management of Differentiated Thyroid Cancer in Pregnancy
}

\author{
Syed Ali Imran ${ }^{1}$ and Murali Rajaraman ${ }^{2}$ \\ ${ }^{1}$ Division of Endocrinology and Metabolism, Dalhousie University, Halifax, NS, Canada B3H 2 Y9 \\ ${ }^{2}$ Department of Radiation Oncology, Dalhousie University, Halifax, NS, Canada B3H 2 Y 9
}

Correspondence should be addressed to Syed Ali Imran, ali.imran@dal.ca

Received 23 March 2011; Accepted 31 March 2011

Academic Editor: Bijay Vaidya

Copyright (C 2011 S. A. Imran and M. Rajaraman. This is an open access article distributed under the Creative Commons Attribution License, which permits unrestricted use, distribution, and reproduction in any medium, provided the original work is properly cited.

In young women, differentiated thyroid cancer is the second most common malignancy diagnosed around the time of pregnancy. Management of thyroid cancer during pregnancy poses distinct challenges due to concerns regarding maternal and fetal wellbeing. In most cases surgery can be safely delayed until after delivery and with adequate management and outcome of pregnancy in women with thyroid cancer is excellent. Ideally these patients should be managed by a multidisciplinary team, and management plan should be determined by a consensus between the patient and the healthcare team.

\section{Introduction}

With the rising incidence of differentiated thyroid cancer (DTC), particularly in younger women, DTC is the second most common cancer diagnosed around the time of pregnancy with a prevalence of 14 per 100,000 [1]. Normal physiological changes occurring during pregnancy and concerns regarding fetal well-being pose distinct challenges to all aspects of DTC management. This paper reviews various facets of DTC management during pregnancy based on the published evidence and extensive clinical experience of the authors.

\section{Is Pregnancy a Risk Factor for Thyroid Cancer?}

Since DTC has a threefold higher incidence in women of reproductive age [2], an association between estrogen, human chorionic gonadotropin (HCG), and DTC has long been speculated. Several studies have suggested an association between the risk of DTC and high parity $[3,4]$, and there is also evidence that use of fertility agent, clomiphene, in parous women is associated with a higher risk of DTC [5]. The data regarding an association between estrogen and DTC, however, are inconsistent, with some studies reporting a pro-proliferative effect of estrogen on thyroid cancer cell lines [6], while others showing a stimulatory effect of estrogen on normal and adenomatous thyroid only, but not on thyroid cancer [7]. The clinical data are also conflicting; one study reported a higher risk of DTC in women exposed to estrogen-containing oral contraceptive and postmenopausal hormone replacement therapy [8], while another study reported no association between the use of exogenous estrogens and DTC [9]. Similar discordance exists in data regarding the outcome of DTC diagnosed during pregnancy; for instance, one study suggests that DTC diagnosed during pregnancy is associated with poorer prognosis and is more likely to have positive ER $\alpha$ expression as compared to tumours diagnosed in nongravidic period [10], while another retrospective study comparing the outcome of DTC diagnosed in pregnant women with age-matched controls showed no significant difference in cancer recurrence or cancer-related death [11]. The data regarding the effect of HCG on DTC are also nonconfirmatory. Although rising HCG during pregnancy has a stimulatory effect on thyroid hormone production, there is no evidence to date linking HCG with DTC. In a large cohort of women treated with fertility drugs, the use of HCG was not associated with a higher risk of DTC [5]. In summary, therefore, epidemiologic data suggest an 
association between high parity and risk of DTC; but there is lingering unclarity regarding the outcome of DTC that is diagnosed during pregnancy.

\section{Management of Thyroid Cancer during Pregnancy}

The management of DTC during pregnancy generally falls into two clinical scenarios. One includes those women who are diagnosed de novo with DTC during pregnancy, while the other includes women with previous history of DTC who have either become pregnant or are planning pregnancy. Both groups present distinct therapeutic challenges requiring specific clinical approach based on disease stage, patient preference, and stage of pregnancy.

3.1. Thyroid Surgery during Pregnancy. For most women with newly diagnosed DTC or those with resectable macroscopic recurrence, a decision about whether or not to perform surgery during pregnancy has to be made. This question is perhaps the biggest source of vexation for patients and physicians alike. So far there has been no prospective study comparing the outcome of DTC in women undergoing surgery during pregnancy versus those where surgery was delayed until after delivery. A retrospective, cross-sectional study comparing 201 pregnant women undergoing thyroid and parathyroid surgery with age-matched nonpregnant controls reported that pregnant women had significantly longer hospital stay, higher hospital costs, and higher rates of general and endocrine complications [12]. Another large survey of almost thirteen thousand pregnant women reported a significantly higher risk of spontaneous abortion in women who underwent surgery during gestation compared with those who did not have surgery [13]. However, the risk of surgery during pregnancy must be balanced against patients' anxiety and perceived concern of tumour growth in case surgery is delayed for several months. This question was addressed through a retrospective study [11] that compared outcomes of DTC in 61 pregnant women with 528 agematched nonpregnant controls. Of pregnant women with DTC, one underwent surgery in the first trimester, twelve in the second, and one in the third trimester, while most of the patients underwent surgery after delivery. After a median followup of 22.4 years, no significant differences in recurrence were observed between women who underwent surgery during or after pregnancy.

Currently there is no consensus about the optimum timing of surgery for DTC in pregnancy [14], and individualized decisions are generally based on patients' wishes and other risk factors, though most would agree that, in the absence of aggressive disease, it is reasonable to delay surgery until after delivery [15]. On the other hand, if surgery is to be considered, for example, in case of large tumour, compressive symptoms, aggressive pathological or clinical features, rapid enlargement of tumor, or patients' concern, it should be performed in the second trimester before 24-week gestation [14] primarily due to an increased risk of spontaneous abortion when surgery is performed in the first trimester [13].

In our interdisciplinary clinic setting (attended by an endocrinologist, surgeon, and a radiation oncologist), we stratify individual patient-risk based on several factors such as cytological features (in case of newly identified DTC), pathological aggressiveness and previous tumor behavior (in case of recurrence), rapidity of growth, compressive symptoms, and ultrasound features while also taking into account patient's wishes and concerns and the obstetrician's opinion before reaching a consensus. In the absence of any high-risk features, we would normally prefer to delay surgery until after delivery but we closely monitor patients throughout pregnancy by performing neck ultrasound scans during each trimester.

3.2. Radioiodine Therapy and Pregnancy. Radioiodine $\left({ }^{131} \mathrm{I}\right)$ administration during pregnancy is contraindicated due to the sequelae of exposing the embryo or fetus to high doses of radiation which include fetal hypothyroidism, attention deficit disorders, memory impairment, mental retardation, malformations, growth changes, induction of malignancies including leukemia, and lethal changes [16]. Women scheduled to have radioiodine therapy should exclude pregnancy with appropriate testing beforehand [14].

${ }^{131}$ I should not be given to nursing women [14] due to the significant accumulation of ${ }^{131} \mathrm{I}$ in the lactating breast and its excretion in breast milk [16]. As most thyroid cancers are slow growing, delaying ${ }^{131} \mathrm{I}$ therapy to allow breastfeeding for a short duration may be considered through discussions between the patient and the treating physician. Postpartum ${ }^{131}$ I therapy should be deferred for at least 6-8 weeks after lactating women have stopped breastfeeding [14]. There is a paucity of reliable data on the kinetics of ${ }^{131} \mathrm{I}$ excretion in breast milk. Therefore, after ${ }^{131} \mathrm{I}$ therapy, it is recommended that breastfeeding should only be resumed with the birth of another child [17, 18]. In order to avoid stagnation of ${ }^{131} \mathrm{I}$ in the lactating breast and minimize the risk of breast radiation exposure, suppression of lactation through dopaminergic agents has been utilized but this should only be used very cautiously [14] after discussion with the patient. Although one large study suggested a possible increase in miscarriage rate if conception occurred within 6 months of ${ }^{131}$ I therapy [19], subsequent studies failed to confirm adverse outcomes for pregnancies or offspring related to previous ${ }^{131}$ I therapy [20]. A conservative recommendation is that women receiving radioiodine therapy should avoid pregnancy for 6-12 months [14] to prevent any increase in risk of infertility, miscarriage, or fetal malformation [21].

\subsection{Thyroid Hormone Replacement during Pregnancy. Most} women undergoing subtotal or total thyroidectomy for DTC require thyroid hormone replacement. Adequate thyroid hormone levels are crucial for maternal and fetal wellbeing, and several studies have reported that even mild hypothyroidism during pregnancy is associated with both adverse maternal and fetal outcomes. For instance, one study showed that children of women with undiagnosed 
hypothyroidism during pregnancy had lower IQ score than their age-matched controls [22]. In another study of women without overt thyroid dysfunction, the risk of miscarriage, fetal or neonatal death increased by $60 \%$ with every doubling in TSH concentration [23]. However, the data regarding low TSH in pregnancy is relatively reassuring, and, in a large survey of 25,765 women of which 433 had subclinical hyperthyroidism, low TSH was not associated with adverse outcomes [24].

The two major challenges in assessing and replacing thyroid hormones during pregnancy are emulating various physiological changes occurring in the thyroid gland during pregnancy and limitations of the commonly utilized laboratory tests for testing thyroid function. The thyroid gland undergoes remarkable changes during pregnancy. A rising HCG in early pregnancy, due to its similarity to thyroid-stimulating hormone (TSH), promotes the release of thyroid hormones which consequently leads to a transient drop in serum TSH values. At the same time, an increasing estrogen level causes two- to threefold rise in thyroid-binding globulin which alters the measured levels of total thyroxine (T4) and triiodothyronine (T3) and to some extent free thyroid hormones as well [25], thus limiting the usefulness of thyroid hormone measurement. This is further complicated by the fact that there can be a wide interassay variability in measured thyroid hormones during pregnancy [26]. Several other factors such as gestational age and singleton versus multiple-birth pregnancy can also alter thyroid hormone levels, in particular serum TSH values [27, 28]. A large survey of over thirteen thousand pregnant women reported a much tighter reference range for TSH especially in early pregnancy (2.5th and 95th percentiles of $0.1 \mathrm{mIU} / \mathrm{L}$ and $2.5 \mathrm{mIU} / \mathrm{L}$, resp.), as compared with general population [27]. Based on these physiological variations, it is ideal to use gestational age-specific reference ranges expressed as multiples of the median, instead of reference values based on general population; however, most commercial assays do not quote pregnancy-specific reference values. Recently more elaborate techniques such as liquid chromatography-tandem mass spectrometry and equilibrium dialysis have been utilized to assess serum T4 in pregnancy $[29,30]$, but, apart from being much more expensive and time consuming, the correlation between free T4 measured through these techniques and serum TSH in pregnancy remains poor [29]. Furthermore, the requirement for thyroid hormone replacement increases by as much as $20-40 \%$ during pregnancy starting as early as the first few weeks of gestation $[31,32]$. Due to its long halflife, T4 administration can take as much as $4-6$ weeks before reaching a steady state in plasma.

With these multiple factors, pursuing a narrow therapeutic TSH target during pregnancy can be quite challenging. One study looked at the effect of empirically increasing the dose of thyroxin replacement immediately upon confirmation of pregnancy and concluded that giving an extra two tablets of thyroxin each week significantly reduces the risk of maternal hypothyroidism during the first trimester and mimics the normal physiology [33]. One caveat with this study was that patients who were athyreotic, those requiring a prepregnancy thyroxin dose of at least $100 \mu \mathrm{g} / \mathrm{d}$, and those with prepregnancy serum TSH concentrations below $1.5 \mathrm{mIU} /$ liter had the highest risk for developing TSH suppression and required subsequent dose modifications after the initial intervention.

In our centre, whenever possible, we typically begin the management of these patients with proper prepregnancy counseling by informing our patients about the rationale for more frequent TSH testing and the need for dose adjustment. In addition, we make patients aware of the possibility of reduced thyroxin absorption with commonly used prepregnancy supplements such as iron and calcium and advise to take them separately from their thyroxin. Those who are already taking suppressive TSH therapy and are planning to get pregnant are typically advised to reduce the dose of thyroxin aiming for a TSH in the range of 0.5$2.5 \mathrm{mIU} / \mathrm{L}$. Upon confirmation of pregnancy, the dose is increased by an additional two tablets each week if TSH is $\geq 1.5 \mathrm{mIU} / \mathrm{L}$ and by one tablet if TSH is $<1.5 \mathrm{mIU} / \mathrm{L}$. Serum TSH levels are checked every $4-6$ weeks and the dose adjusted to achieve and maintain TSH in the range of $0.5-2.5 \mathrm{mIU} / \mathrm{L}$ during pregnancy.

3.4. Followup of Thyroid Cancer during Pregnancy. Most pregnant women with low-risk DTC require little more than routine TSH monitoring and periodic clinical examination during pregnancy. Radioactive iodine scan or stimulated thyroglobulin $(\mathrm{Tg})$ estimation through either thyroid hormone withdrawal or recombinant TSH (Thyrogen) is not justifiable in pregnancy. Several studies have reported that although serum $\mathrm{Tg}$ levels can vary significantly during each trimester, the overall values remain well within the normal nonpregnant range [34-36].

In our centre, we devise our followup strategy for such patients based on their risk factors. Pregnant women with low-risk DTC who were regarded free of disease prior to pregnancy, aside from their thyroxin dose adjustment, are followed on a three monthly (once in each trimester) basis with an unstimulated $\mathrm{Tg}$, and a thorough neck examination is conducted at each visit. Those women who have highrisk DTC or had documented recurrence of DTC prior to pregnancy are followed more rigorously on a three monthly basis with unstimulated $\mathrm{Tg}$ and neck ultrasonography. Normal reference ranges for serum $\mathrm{Tg}$ are irrelevant for followup of such patients, and decision regarding cancer progress is based on their prepregnancy Tg levels as well as ultrasonography findings.

\section{The Role of Multidisciplinary Team in Management of Thyroid Cancer}

Outside specialist centers, DTC patients have traditionally been managed by a variety of specialties which leads to an inconsistent and fragmented care, borne out by several studies from various centers [37-39]. Patient surveys have also confirmed poor and inconsistent coordination of thyroid cancer care among different caregivers [40]. Over the past decade, several centers of excellence have developed models of multidisciplinary teams comprising 
surgeons, radiologists, pathologists, endocrinologists, and allied specialists to deliver coordinated care within hospitals which ensures that each individual patient gets appropriate treatment decision made by a team of experts [41]. In our centre, all DTC patients (including pregnant females) are assessed and followed by a team of specialists including a surgeon, an endocrinologist, a radiation oncologist, a dietitian, specialist nurses and, in case of pregnant women, the team works closely with an obstetrician and gynecologist. In our opinion, pregnant women with DTC should ideally be referred to a specialist centre but, in the absence of such facility, management decisions should be made through close cooperation of all caregivers and the patient.

\section{References}

[1] L. H. Smith, B. Danielsen, M. E. Allen, and R. Cress, "Cancer associated with obstetric delivery: results of linkage with the California cancer registry," American Journal of Obstetrics and Gynecology, vol. 189, no. 4, pp. 1128-1135, 2003.

[2] A. Jemal, R. Siegel, E. Ward, T. Murray, J. Xu, and M. J. Thun, "Cancer statistics, 2007," CA: A Cancer Journal for Clinicians, vol. 57, no. 1, pp. 43-66, 2007.

[3] O. Kravdal, E. Glattre, and T. Haldorsen, "Positive correlation between parity and incidence of thyroid cancer: new evidence based on complete Norwegian birth cohorts," International Journal of Cancer, vol. 49, no. 6, pp. 831-836, 1991.

[4] S. Preston-Martin, L. Bernstein, and M. C. Pike, "Thyroid cancer among young women related to prior thyroid disease and pregnancy history," British Journal of Cancer, vol. 55, no. 2, pp. 191-195, 1987.

[5] C. G. Hannibal, A. Jensen, H. Sharif, and S. K. Kjaer, "Risk of thyroid cancer after exposure to fertility drugs: results from a large Danish cohort study," Human Reproduction, vol. 23, no. 2, pp. 451-456, 2008.

[6] M. L. Lee, G. G. Chen, A. C. Vlantis, G. M. K. Tse, B. C. H. Leung, and C. A. Van Hasselt, "Induction of thyroid papillary carcinoma cell proliferation by estrogen is associated with an altered expression of Bcl-xL," Cancer Journal, vol. 11, no. 2, pp. 113-121, 2005.

[7] L. Del Senno, E. Degli Uberti, S. Hanau, R. Piva, R. Rossi, and G. Trasforini, "In vitro effects of estrogen on tgb and c-myc gene expression in normal and neoplastic human thyroids," Molecular and Cellular Endocrinology, vol. 63, no. 1-2, pp. 6774, 1989.

[8] A. M. McTiernan, N. S. Weiss, and J. R. Daling, "Incidence of thyroid cancer in women in relation to reproductive and hormonal factors," American Journal of Epidemiology, vol. 120, no. 3, pp. 423-435, 1984.

[9] W. J. Mack, S. Preston-Martin, L. Bernstein, D. Qian, and M. Xiang, "Reproductive and hormonal risk factors for thyroid cancer in Los Angeles County females," Cancer Epidemiology Biomarkers and Prevention, vol. 8, no. 11, pp. 991-997, 1999.

[10] G. Vannucchi, M. Perrino, S. Rossi et al., "Clinical and molecular features of differentiated thyroid cancer diagnosed during pregnancy," European Journal of Endocrinology, vol. 162 , no. 1, pp. 145-151, 2010.

[11] M. Moosa and E. L. Mazzaferri, "Outcome of differentiated thyroid cancer diagnosed in pregnant women," Journal of Clinical Endocrinology and Metabolism, vol. 82, no. 9, pp. 2862-2866, 1997.
[12] S. Kuy, S. A. Roman, R. Desai, and J. A. Sosa, "Outcomes following thyroid and parathyroid surgery in pregnant women," Archives of Surgery, vol. 144, no. 5, pp. 399-406, 2009.

[13] J. B. Brodsky, E. N. Cohen, and B. W. Brown, "Surgery during pregnancy and fetal outcome," American Journal of Obstetrics and Gynecology, vol. 138, no. 8, pp. 1165-1167, 1980.

[14] D. S. Cooper, G. M. Doherty, B. R. Haugen et al., "Revised American thyroid association management guidelines for patients with thyroid nodules and differentiated thyroid cancer," Thyroid, vol. 19, no. 11, pp. 1167-1214, 2009.

[15] R. P. Owen, K. J. Chou, C. E. Silver et al., "Thyroid and parathyroid surgery in pregnancy," European Archives of OtoRhino-Laryngology, vol. 267, pp. 1825-1835, 2010.

[16] C. A. Gorman, "Radioiodine and pregnancy," Thyroid, vol. 9, no. 7, pp. 721-726, 1999.

[17] E. B. Silberstein, A. Alavi, H. R. Balon et al., "Society of Nuclear Medicine Procedure Guideline for Therapy of Thyroid Disease with Iodine-131 (Sodium Iodide) Version 2.0," http://interactive.snm.org/index.cfm?PageID=805.

[18] "Release of patients administered radioactive material," US nuclear regulatory commission, Regulatory Guide 8.39, April 1997, http://www.nucmed.com.

[19] M. Schlumberger, F. De Vathaire, C. Ceccarelli et al., "Exposure to radioactive iodine-131 for scintigraphy or therapy does not preclude pregnancy in thyroid cancer patients," Journal of Nuclear Medicine, vol. 37, no. 4-6, pp. 606-612, 1996.

[20] J. P. Garsi, M. Schlumberger, C. Rubino et al., "Therapeutic administration of I for differentiated thyroid cancer: radiation dose to ovaries and outcome of pregnancies," Journal of Nuclear Medicine, vol. 49, no. 5, pp. 845-852, 2008.

[21] E. H. Holt, "Care of the pregnant thyroid cancer patient," Current Opinion in Oncology, vol. 22, no. 1, pp. 1-5, 2010.

[22] J. E. Haddow, G. E. Palomaki, W. C. Allan et al., "Maternal thyroid deficiency during pregnancy and subsequent neuropsychological development of the child," New England Journal of Medicine, vol. 341, no. 8, pp. 549-555, 1999.

[23] N. Benhadi, W. M. Wiersinga, J. B. Reitsma, T. G. M. Vrijkotte, and G. J. Bonsel, "Higher maternal TSH levels in pregnancy are associated with increased risk for miscarriage, fetal or neonatal death," European Journal of Endocrinology, vol. 160, no. 6, pp. 985-991, 2009.

[24] B. M. Casey, J. S. Dashe, C. E. Wells, D. D. McIntire, K. J. Leveno, and F. G. Cunningham, "Subclinical hyperthyroidism and pregnancy outcomes," Obstetrics and Gynecology, vol. 107, no. 2, pp. 337-341, 2006.

[25] R. H. Lee, C. A. Spencer, J. H. Mestman et al., "Free T4 immunoassays are flawed during pregnancy," American Journal of Obstetrics and Gynecology, vol. 200, no. 3, pp. 260.e1-260.e6, 2009.

[26] E. Berta, L. Samson, A. Lenkey et al., "Evaluation of the thyroid function of healthy pregnant women by five different hormone assays," Pharmazie, vol. 65, no. 6, pp. 436-439, 2010.

[27] J. S. Dashe, B. M. Casey, C. E. Wells et al., "Thyroidstimulating hormone in singleton and twin pregnancy: importance of gestational age-specific reference ranges," Obstetrics and Gynecology, vol. 106, no. 4, pp. 753-757, 2005.

[28] J. E. Haddow, G. J. Knight, G. E. Palomaki, M. R. McClain, and A. J. Pulkkinen, "The reference range and within-person variability of thyroid stimulating hormone during the first and second trimesters of pregnancy," Journal of Medical Screening, vol. 11, no. 4, pp. 170-174, 2004.

[29] J. Jonklaas, N. Kahric-Janicic, O. P. Soldin, and S. J. Soldin, "Correlations of free thyroid hormones measured by 
tandem mass spectrometry and immunoassay with thyroidstimulating hormone across 4 patient populations," Clinical Chemistry, vol. 55, no. 7, pp. 1380-1388, 2009.

[30] B. Yue, A. L. Rockwood, T. Sandrock, S. L. La’ulu, M. M. Kushnir, and A. W. Meikle, "Free thyroid hormones in serum by direct equilibrium dialysis and online solid-phase extractionliquid chromatography/tandem mass spectrometry," Clinical Chemistry, vol. 54, no. 4, pp. 642-651, 2008.

[31] E. K. Alexander, E. Marqusee, J. Lawrence, P. Jarolim, G. A. Fischer, and P. R. Larsen, "Timing and magnitude of increases in levothyroxine requirements during pregnancy in women with hypothyroidism," New England Journal of Medicine, vol. 351, no. 3, pp. 241-310, 2004.

[32] S. J. Mandel, P. R. Larsen, E. W. Seely, and G. A. Brent, "Increased need for thyroxine during pregnancy in women with primary hypothyroidism," New England Journal of Medicine, vol. 323, no. 2, pp. 91-96, 1990.

[33] L. Yassa, E. Marqusee, R. Fawcett, and E. K. Alexander, "Thyroid hormone early adjustment in pregnancy (The THERAPY) trial," Journal of Clinical Endocrinology and Metabolism, vol. 95, no. 7, pp. 3234-3241, 2010.

[34] O. P. Soldin, R. E. Tractenberg, J. G. Hollowell, J. Jonklaas, N. Janicic, and S. J. Soldin, "Trimester-specific changes in maternal thyroid hormone, thyrotropin, and thyroglobulin concentrations during gestation: trends and associations across trimesters in iodine sufficiency," Thyroid, vol. 14, no. 12, pp. 1084-1090, 2004.

[35] Y. Hara, T. Tanikawa, and Y. Sakatsume, "Decreased serum thyroglobulin levels in the late stage of pregnancy," Acta Endocrinologica, vol. 113, no. 3, pp. 418-423, 1986.

[36] K. Kamikubo, T. Komaki, S. Nakamura, S. Sakata, K. Yasuda, and K. Miura, "Theoretical consideration of the effects of dilution on estimates of free thyroid hormones in serum," Clinical Chemistry, vol. 30, no. 5, pp. 634-636, 1984.

[37] S. A. Hundahl, I. D. Fleming, A. M. Fremgen, and H. R. Menck, "A National Cancer Data Base report on 53,856 cases of thyroid carcinoma treated in the U.S., 1985-1995," Cancer, vol. 83, no. 12, pp. 2638-2648, 1998.

[38] M. P. J. Vanderpump, L. Alexander, J. H. B. Scarpello, and R. N. Clayton, "An audit of the management of thyroid cancer in a district general hospital," Clinical Endocrinology, vol. 48, no. 4, pp. 419-424, 1998.

[39] S. Hölzer, C. Reiners, K. Mann et al., "Patterns of care for patients with primary differentiated carcinoma of the thyroid gland treated in Germany during 1996," Cancer, vol. 89, no. 1, pp. 192-201, 2000.

[40] http://www.thyroid-cancer-alliance.org.

[41] "Improving Outcomes in Head and Neck Cancer," The Manual, National Institute of Clinical Excellence, November 2004, http://www.nice.org.uk. 


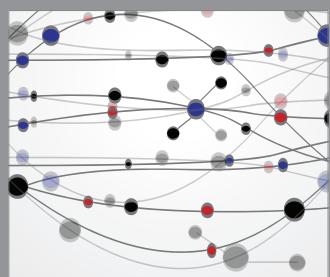

The Scientific World Journal
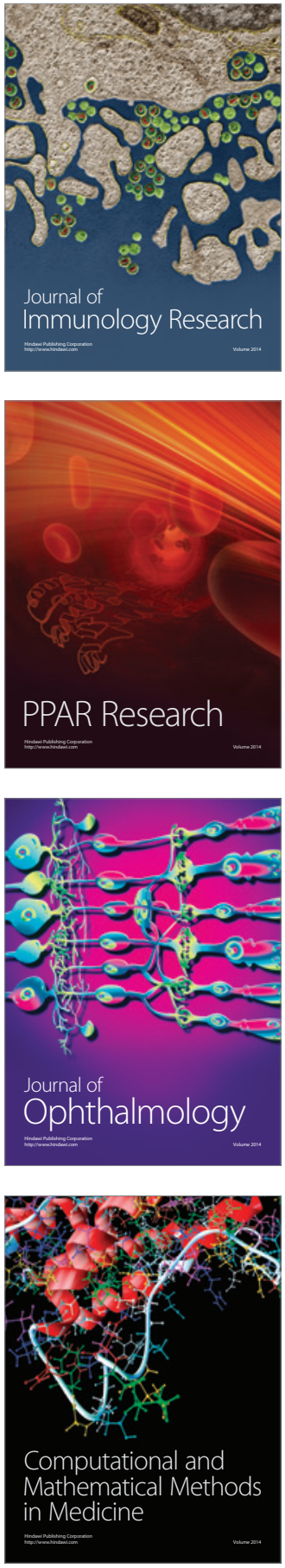

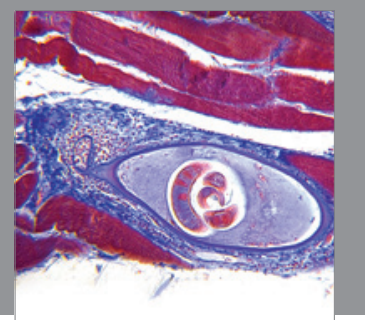

Gastroenterology

Research and Practice
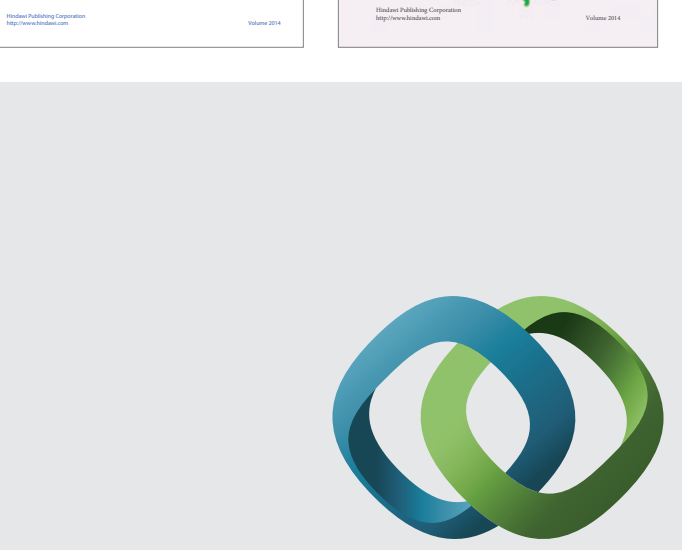

\section{Hindawi}

Submit your manuscripts at

http://www.hindawi.com
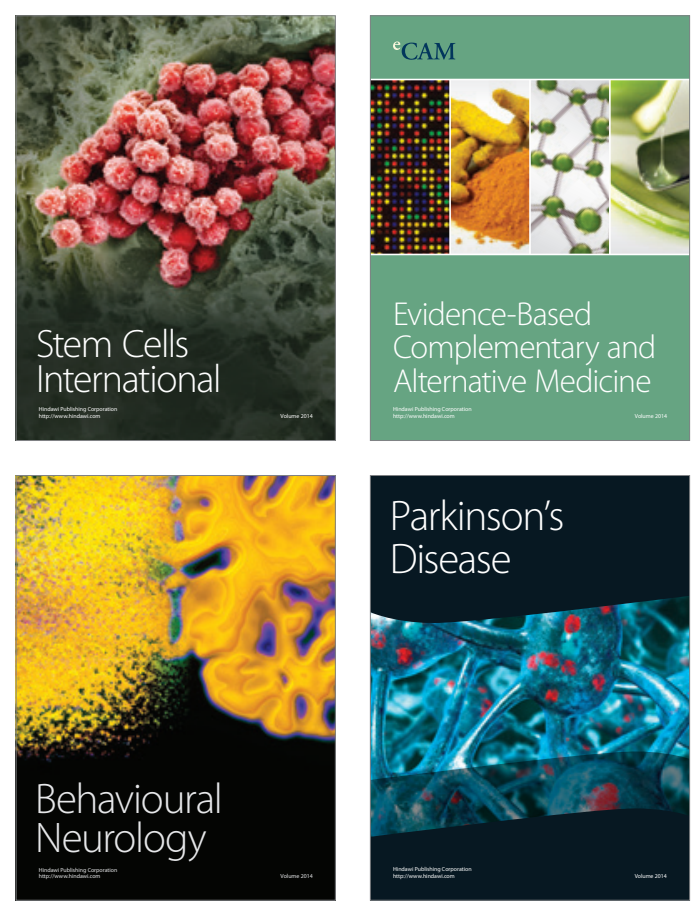

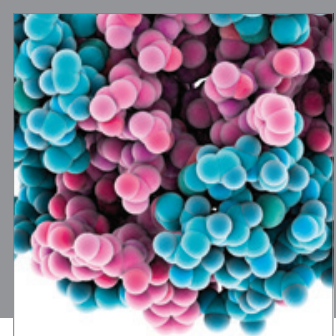

Journal of
Diabetes Research

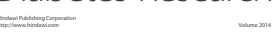

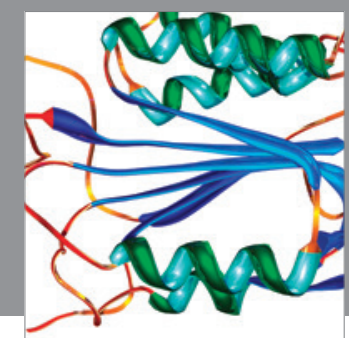

Disease Markers
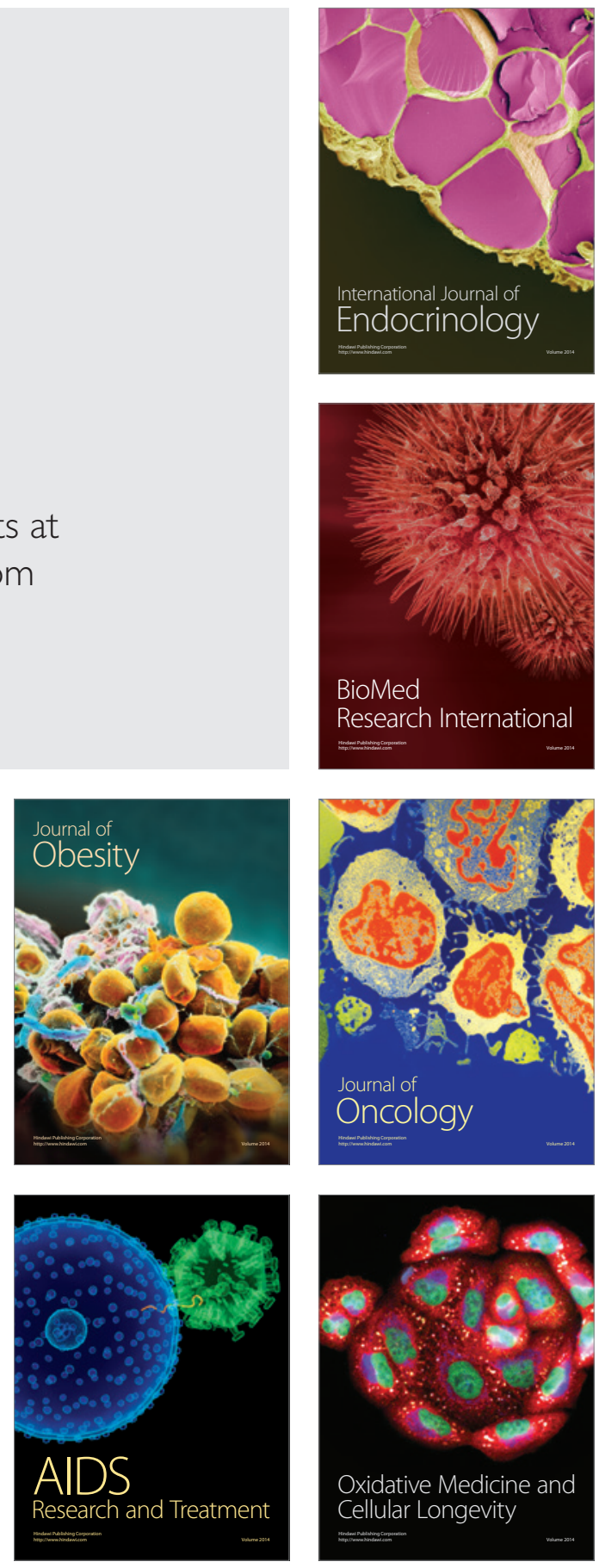